\title{
Análise dos discursos das juventudes sobre saúde auditiva dialogados na web rádio
}

\author{
Analysis of youth's discourses about hearing health dialogued on web radio \\ Análisis de los discursos de los jóvenes sobre los diálogos de salud auditiva en la web radio
}

Recebido: 03/06/2021 | Revisado: 13/06/2021 | Aceito: 15/06/2021 | Publicado: 30/06/2021

\author{
Edine Dias Pimentel Gomes \\ ORCID: https://orcid.org/0000-0001-5990-6358 \\ Universidade Estadual do Ceará, Brasil \\ E-mail: edinemc@hotmail.com \\ Raimundo Augusto Martins Torres \\ ORCID: https://orcid.org/0000-0002-8114-4190 \\ Universidade Estadual do Ceará, Brasil \\ E-mail: augusto.torres@uece.br \\ Karlla da Conceição Bezerra Brito Veras \\ ORCID: https://orcid.org/0000-0001-7464-1992 \\ Universidade Estadual do Ceará, Brasil \\ E-mail: karlla_veras@hotmail.com \\ Isabela Gonçalves Costa \\ ORCID: https://orcid.org/0000-0002-9972-8258 \\ Universidade Estadual do Ceará, Brasil \\ E-mail: isabela.goncalves@aluno.uece.br \\ Luna Morgana de Oliveira Moraes \\ ORCID: https://orcid.org/0000-0003-0413-1414 \\ Universidade Estadual do Ceará, Brasil \\ E-mail: luna.morgana @aluno.uece.br \\ Breno da Silva Albano \\ ORCID: https://orcid.org/0000-0002-6030-5178 \\ Universidade Estadual do Ceará, Brasil \\ E-mail: breno.albano@aluno.uece.br
}

\section{Resumo}

Objetivo: analisar os discursos das juventudes produzidos no Programa "Em Sintonia com a Saúde", veiculado na web rádio, acerca de diálogos sobre saúde auditiva. Metodologia: pesquisa descritiva, do tipo documental, com abordagem qualitativa, abrangendo o conteúdo da comunicação síncrona, a partir de uma amostra de 83 participantes. Resultados: as juventudes demonstram em seus discursos que os saberes das ciências modernas compõem seus repertórios de conhecimento e interesses sobre o tema, bem como suas dizibilidades circularam em torno dos cuidados higiênicos, das regulações legais com a sonoridade pública e privada, dos usos adequado dos dispositivos auriculares e dos adoecimentos causados pelo excesso de sonoro. Conclusão: Em vista disso, os diálogos mediados na web rádio fortaleceram os cuidados com a saúde auditiva, facilitados pelo uso de informações acessadas através dos dispositivos de comunicação digitais, essenciais a promoção da saúde.

Palavras-chave: Surdez; Adolescente; Fonoaudiologia; Educação em saúde; Promoção da saúde; Tecnologia da informação.

\begin{abstract}
Objective: to analyze the youth's speeches produced in the Program "In Tuning with Health", broadcast on web radio, about dialogues on hearing health. Methodology: descriptive and documentary research, with qualitative approach, covering the content of synchronous communication, from a group of 83 participants. Results: the youths demonstrate in their speeches that the knowledge of modern sciences make up their repertoire of knowledge and interests on the subject, as well as their sayings circulated around hygienic care, legal regulations with public and private sound, the proper uses of ear devices and illnesses caused by excessive noise. Conclusion: In view of this, dialogues mediated from web radio strengthened hearing health care, facilitated by the use of information accessed through digital communication devices, essential to health promotion.
\end{abstract}

Keywords: Deafness; Adolescent; Speech, language and hearing sciences; Health education; Health promotion; Information technology.

\section{Resumen}

Objetivo: analizar los discursos de jóvenes producidos en el programa "En sintonía con la salud", transmitido por radio web, sobre diálogos sobre salud auditiva. Metodología: investigación descriptiva, tipo documental, con enfoque cualitativo, cubriendo el contenido de la comunicación sincrónica, a partir de una muestra de 83 participantes. 
Resultados: los jóvenes demuestran en sus discursos que los conocimientos de las ciencias modernas conforman su repertorio de conocimientos e intereses sobre el tema, así como sus refranes circulados en torno al cuidado higiénico, normativas legales con sonido público y privado, los usos adecuados de los dispositivos auditivos. y enfermedades causadas por ruido excesivo. Conclusión: Ante esto, los diálogos radiales en la web fortalecieron la atención de la salud auditiva, facilitada por el uso de información a la que se accede a través de dispositivos de comunicación digital, fundamental para la promoción de la salud.

Palabras clave: Sordera; Adolescente; Fonoaudiología; Educación en Salud; Promoción de la salud; Tecnología de la información.

\section{Introdução}

No Brasil, as políticas públicas de Saúde Auditiva são desempenhadas através de práticas de cuidado, instituídas desde o nascimento, possibilitando o diagnóstico de deficiências e o direcionamento de cuidados necessários, ainda na infância, bem como o acompanhamento do desenvolvimento infanto-juvenil, de modo a avaliar a acuidade auditiva e demandas quanto ao uso de dispositivos auditivos (Vieira et al., 2015; Jardim et al. 2017).

Por conseguinte, a saúde auditiva, quando não abordada de forma precoce, pode acarretar uma série de prejuízos no desenvolvimento vital do ser humano, dada possibilidade de crescimento e desenvolvimento social com deficiência na audição. Nesse contexto, a instituição de práticas de educação em saúde por profissionais assistenciais da primeira infância possibilita a compreensão de demandas em saúde auditiva dos escolares, viabilizando a avaliação precoce, além de experimentações de pesquisas e estudos que integram a formação contínua (Jacob et al., 2019).

Nesse intento, o uso das Tecnologias Digitais de Informação e Comunicação (TDIC), auxiliam na ampliação e eficácia das trocas de saberes e alcance das juventudes escolares, em virtude da acessibilidade dos canais de comunicação e cultura digital. Assim, as TDIC constituem instrumentos introduzidos na cultura da sociedade, através de diversos desenhos e modos de utilidade, a partir de dispositivos como tablets, celulares e computadores, comuns as atividades humanas de vida social (Costa et al., 2015).

Com efeito, o processo de aprendizagem, constituído no ciberespaço como ubíquo, pode ocorrer em qualquer lugar, desde que haja acesso aos dispositivos digitais, dispensando o deslocamento geográfico, visto que a informação é disponibilizada de forma livre e contínua (Santaella, 2014), criando redes de comunicação dialógicas, bem como novos modos de aprender, ensinar e estimular o conhecimento. No que concerne à saúde, o acesso à informação constitui um direito garantido pela constituição federal.

Atualmente, a comunicação no ciberespaço da internet se apresenta em diferentes formatos, tais como as web rádios, que são, entre inúmeros outros megafones, lugares de fala e interações linguísticas produzidas por usuários, possibilitando a produção de conteúdo educacional, aliada a promoção de práticas de web cuidado em saúde, mediadas no ciberespaço (Torres et al., 2015).

Portanto, a utilização das TDIC auxiliam a produção de conhecimentos pelos usuários, por meio do uso de dispositivos digitais, apensados aos espaço de conteúdo das web rádios, oportunizando a prestação de serviço à comunidade, através da extensão da comunicação em saúde e de outras temas, transpondo barreiras físicas dos espaço de produção do conhecimento científico e acadêmico nas universidades, a partir do estabelecimento de diálogos e troca de conhecimentos com a sociedade, efetivando, assim, uma das funções essenciais destas instituições de formação superior (Ferreira \& Freitas, 2018).

Nesse sentido, o Programa "Em Sintonia com a Saúde - S@S”, da Web Rádio AJIR, vinculada à Universidade Estadual do Ceará (UECE), constitui um ambiente digital de mediação da comunicação e educação em saúde com as juventudes escolares, universitárias e com os internautas que habitam cotidianamente a rede mundial de computadores.

Assim, a experiência dos diálogos com as juventudes, oportunizados pela web rádio, fomentaram a seguinte indagação: “Como as juventudes surdas, em diálogo com a web rádio, produzem práticas discursivas de cuidado de si?". Por 
conseguinte, a questão de pesquisa conecta-se com o objetivo do estudo, que consiste na análise dos discursos produzidos no Programa “Em Sintonia com a Saúde - S@S” com as juventudes surdas sobre saúde auditiva.

\section{Metodologia}

\subsection{Tipo ou delineamento do estudo}

Pesquisa descritiva, do tipo documental, com abordagem qualitativa, realizada com 83 jovens que participaram do Programa: “Em Sintonia com a Saúde - S@S”, com o tema saúde auditiva, veiculado pela Web Rádio AJIR, em 2019.

Para Minayo (2010), a abordagem qualitativa centra-se na experiência humana atribuída de significados derivados dos contextos da vida, promovendo aproximação fundamental e de intimidade entre sujeito e objeto, envolvendo com empatia aos motivos, às intenções, aos projetos dos atores, a partir dos quais as ações, as estruturas e as relações tornam-se significativas.

\subsection{Cenário da coleta de dados}

A produção do programa foi realizada em um estúdio localizado na Universidade Estadual do Ceará (Fortaleza, CE, Brasil) e exibido através da plataforma YouTube, possibilitando a interação dos participantes e envio de perguntas através dos grupos de WhatsApp, disponibilizados e criados pela web rádio, permitindo uma comunicação dialógica síncrona e ao vivo, de modo que $\mathrm{o}$ apresentador pudesse sanar as perguntas dos jovens que viessem a surgir durante o programa.

A dinâmica do programa prioriza as interações dialógicas entre os profissionais convidados e as juventudes universitárias e escolares inseridas nas quatro grandes regiões de saúde do estado. Contudo, para esta pesquisa, considerou-se as Perguntas-Discursos (PD) dos participantes de uma Instituição de Ensino Superior (IES) e de três escolas públicas da cidade de Sobral (CE). Ademais, compreende-se por PD as práticas discursivas que engendram os modos de viver juvenis e contemporâneos (Torres et al., 2015).

A Web Rádio AJIR tem 13 anos de fundação, sendo exibida através de um canal online, no qual são desenvolvidas ações de promoção do cuidado educativo destinadas ao público juvenil de escolas públicas e universidades do estado. Vale destacar, que os dados desta pesquisa foram coletados anteriormente à pandemia causada pelo novo coronavírus (SARS-CoV2). Assim, a comunicação e interações virtuais já constituíam práticas de web cuidado em saúde, facilitadas com a imersão nos ciberespaços da Internet.

Em 2020 e 2021, a programação da web rádio teve seu conteúdo de comunicação e diálogos centralizados no fortalecimento da rede de proteção social para prevenção da pandemia, bem como a divulgação e compartilhamento dos saberes científicos, sociais e culturais que facilitam o enfrentamento global da COVID-19 (Torres et al., 2020).

Assim, a opção pelo termo juventudes, foi cunhado tendo como referência a compreensão de que as experiências juvenis são produzidas em contextos históricos e sociais diversos, com ou sem composição da mesma faixa de idade, gêneros, classe social, raça-cor, identidades sexuais, entre outros agenciamentos que rostizam seus modos de vida e refletem a cultural vivida em seus cotidianos.

Essas concepções de juventudes plurais na pesquisa expressam as heterogeneidades e diversidades, materializadas nas diversas condições de cada sociedade e/ou grupos étnicos, raciais, nômades, religiosos e na riqueza e complexidade da diversidade sexual e de gêneros, bem como nos diversos espaços geográficos percorridos pela população juvenil (Sales \& Vasconcelos, 2016; Torres et al., 2020).

\subsection{Tratamento e Análise dos dados}

Baseado nisso, foram selecionadas doze PD, nas quais os participantes foram nomeados pela palavrada "Jovem" seguida de um algarismo arábico, a fim de preservar a identificação dos participantes. As perguntas-discursos foram 
organizadas em duas categorias, descritas em: Discursos das Juventudes Escolares (DJE) e Discursos das Juventudes Universitárias (DJU), em virtude das suas conexões temáticas.

A seguir, as PD foram analisadas a partir da sistemática analítica do discurso, descrita por Foucault (1989), considerando os discursos como práticas de saber e poder, com efeito na produção do cuidado de si, que nesta experiência, é apresentada pelo desenho de práticas de comunicação em saúde, referenciadas como web cuidado, dada suas espacialidades "agrimensadas" no ciberespaços, onde as juventudes praticam seus modos de vidas contemporâneos (Torres et al., 2012).

\subsection{Aspectos éticos}

Esta pesquisa teve aprovação do Comitê de Ética em Pesquisa da UECE, sob o Parecer $\mathrm{n}^{\circ}$ 1.761.115/2019, em conformidade com a Resolução 466/2012 do Conselho Nacional de Saúde.

\section{Resultados}

Os discursos produzidos no ciberespaço da web rádio traçam um paralelo com a literatura, no que concerne à temática de saúde auditiva, corroborando com a aplicação e atualização dos saberes prescritivos da ciência moderna, assunto que ganhou alento e lugar de poder com a revolução iluminista do conhecimento no Século XVII (Foucault, 1989).

Neste sentido, reitera-se a força dos saberes das ciências modernas centradas no conhecimento biomédico e na sua aplicabilidade, de acordo com as demandas de saúde humana. No entanto, sua hegemonia e vinculação ao processo produtivo econômico do capital parecem não ter atendido à sua maior amplitude nas necessidades sociais e de saúde ao longo do Século XX e início do Século XXI.

Assim, a sociedade contemporânea alimenta-se da informação e da comunicação, mediada pelo meio digital, possibilitando o desenvolvimento de práticas de cuidado, utilizando os diversos conhecimentos, além de alinhar teoria à prática, estimulando a aprendizagem ubíqua.

Neste sentido, a experiência do uso da web rádio, junto aos diálogos com as juventudes sobre saúde auditiva, vem a problematizar dúvidas e informações seguras sobre o cuidado em saúde, viabilizando dados empíricos para a construção das categorias discursivas a seguir.

\subsection{Discursos das Juventudes Universitárias}

A falta de higiene pode causar a perda auditiva? (JOVEM 1).

As infecções podem causar perda auditiva? (JOVEM 2).

A utilização de cotonetes é prejudicial à saúde auditiva? (JOVEM 3).

A lavagem no ouvido pode levar à perda da audição? (JOVEM 4).

Nota-se que os participantes reconhecem a importância da higiene pessoal, mas temem que os produtos utilizados para a limpeza auricular, amplamente divulgados pela mídia como os mais indicados, possam, de alguma forma, prejudicar a audição.

Quais os cuidados que devemos tomar para proteger a saúde auditiva? (JOVEM 5).

O limite recomendado é de 80 decibéis. Todo barulho acima disso é prejudicial? (JOVEM 6).

O quanto é prejudicial o fone de ouvido? (JOVEM 7).

Quais as principais dúvidas existentes durante uma consulta com o profissional de saúde auditiva? (JOVEM 8). 
As perguntas-discursos dos participantes revelam conhecimentos básicos sobre cuidados com a proteção auditiva, possibilitando reconhecer os limites acerca dessa temática, além da curiosidade sobre as principais atividades exercidas pelo profissional da saúde auditiva durante o atendimento clínico.

Já nos discursos dos participantes escolares, verificamos a atualização do cuidado em saúde auditiva, no que se refere ao uso de dispositivos de auxílio à escuta sonora, apensados aos dispositivos da comunicação digital.

\subsection{Discursos da Juventude Escolares}

Quais fones de ouvido podem ser mais prejudiciais à saúde? (JOVEM 9).

A audição pode ser prejudicada por algo além de barulhos, como uma doença? (JOVEM 10).

Existe alguma lei ou órgão de fiscalização área controlar os ruídos de uma empresa para a segurança do funcionário? (JOVEM 11).

Qual o nível de ruído permitido por lei? (JOVEM 12).

As juventudes apresentam preocupações atuais com relação ao uso cotidianos dos dispositivos auriculares de audição, dadas relações de uso e acesso aos ciberespaços na Internet e demais tecnologias digitais, que apresentam uma gama de "janelas" para interações síncronas e assíncronas atendem as demandas de comunicação digital no cotidiano de suas vidas.

\section{Discussão}

Nos discursos dos participantes foram expressadas, inicialmente, temáticas sobre os cuidados com a higiene auditiva, tais como indagações acerca da limpeza do aparelho auditivo e infecções que possam prejudicá-lo, dificultando o acesso à informação. Contudo, é importante ressaltar que a falta de higiene pode contribuir para a perda auditiva, uma vez que a ausência dessas condições impedem que o organismo consiga atuar de forma orgânica, criando condições para um ambiente de infecções, como o excesso de cerúmen que obstrui o meato acústico externo, onde estão localizadas as glândulas sebáceas e glândulas ceruminosas, interferindo na transmissão do estímulo auditivo (Andrade et al., 2018).

Por conseguinte, as produções discursivas apresentadas pelos Jovens 03 e 04, exteriorizam questionamentos sobre a higienização e o uso de cotonetes, reforçando a necessidade da informação e da comunicação qualificada nesta área, ao mesmo tempo em que reproduzem a centralidade dos saberes das ciências biomédicas. Nesse ínterim, o uso do cotonete não é recomendado, devido a possibilidade de deslocamento do cerúmen substância produzida pelas glândulas sebáceas para lubrificação e proteção do ouvido, até o fundo do Canal Auditivo Externo. Cabe dizer que, excepcionalmente, os excessos desta glândula, quando produzidos em média e alta densidade e com endurecimento do seu conteúdo, podem ser avaliadas e aplicada a técnica de sua retirada por profissionais da saúde habilitados em cuidados clínicos de saúde auditiva (Nunes et al., 2019).

Outrossim, os dizeres das juventudes sobre o cuidado em saúde auditiva são atualizados aos saberes contemporâneos das ciências biológicas, que desde o Século XIX se afirmam como vontades de verdade para gerir o corpo anatomopatológico em inteira conexão com os controles e disciplinamentos do corpo social, seja pela sujeição, resistências e reconfiguração de suas práticas com o cuidado consigo e com suas coletividades. Assim, o corpo, esquadrinhado pelas potências dos saberes médicos a época se ligam aos processos econômicos e sociais para possibilitar a vida viável, pois o corpo individual e o corpo coletivo, geograficamente, deslocados na era medieval, agora se juntam como pelo controle, vigilância e disciplinamento em um corpo conectado a organização social moderna, o estado (Foucault, 1989).

Nas formações discursivas dos Jovens 05 e 06, são visualizados os limites da métrica sonora, representada pelos decibéis ideais para a prevenção de déficits na audição humana. Esta preocupação parece estar relacionada com o ambiente 
educacional, tendo em vista o grande fluxo de pessoas em comunicação e interação verbal e/ou produzindo outros ruídos sonoros.

Neste contexto, a exposição à alta frequência sonoras, como ruídos permanentes e persistentes, igual ou acima de 80 decibéis, podem ocasionar perda auditiva, especialmente no ambiente de trabalho (Pereira \& Teles, 2013), sendo recomendada a utilização de protetores auriculares, ofertados pelas organizações trabalhistas, aliado ao incentivo para seu uso cotidiano como uma ferramenta básica de proteção (Karimi \& Nasiri, 2010; Rocha et al., 2011; Oliveira et al., 2015).

Em vista disso, a proteção auditiva humana repercutiu nos discursos juvenis (Jovem 07 e 08), acerca do uso dos fones de ouvidos, dada sua utilização para hobby, como jogos de videogame e durante o sono, por vezes, com volume elevado, expondo-os aos riscos de perdas auditivas, principalmente a partir do uso prolongado (Widén et al., 2017; Keppler et al., 2015).

Portanto, os diálogos compartilhados pela mediação da comunicação e educação em saúde via web rádio, reforçam os saberes das ciências básicas, os quais ancoram as práticas sociais e clínicas dos profissionais de saúde, especialmente os fonoaudiólogos, incentivando as práticas de cuidado com a saúde, principalmente a auditiva.

Baseado nisso, os participantes (Jovem 09) revelaram interesse em conhecer os tipos de fones de ouvido que podem ser prejudiciais, proporcionando o diálogo com este saber-poder do perito. Em decorrência, foi destacado que o fone intraauricular expõe o usuário a elevados níveis de pressão sonora, uma vez é inserido no canal auditivo externo, ao passo que seu design permite o encaixe adequado na estrutura anatômica do ouvido, impulsionando a elevação do volume para contornar os sons do ambiente externo (Oliveira et al., 2017).

Seguindo este mesmo discurso, as juventudes dialogam sobre as estruturas legais que possibilitam a regulação sonora e como estas apresentam-se no arcabouço jurídico e político brasileiro. Neste campo, a legislação é estabelecida pelos órgãos responsáveis pela verificação das condições adequadas dos ambientes de trabalho, sendo permitido até 80 decibéis, considerado a intensidade e exposição sonora (Gonçalves \& Fontoura, 2018).

Por fim, as juventudes apontam para o desejo de conhecer as doenças provocadas pelo excesso sonoro permanente e com taxas métricas de decibéis acima do regulado pelas organizações do estado. Dentre elas, destaca-se a Perda Auditiva Induzida pelo Ruído (PAIR), que infere problemas neurológicos, digestivos e endócrinos. Por esse motivo, foram estabelecidas legislações trabalhistas, garantindo sua prevenção através de programas de promoção à saúde auditiva nos locais de trabalho (Gonçalves \& Fontoura, 2018). Nesse contexto, a doença de Parkinson, ocasionada pelo comprometimento neurodegenerativo, causa elevação da ansiedade, visto que pessoas com perdas auditivas e/ou surdez têm alteração de suas condições socioemocionais na convivência cotidiana (Silva et al., 2018; Santos \& Silva, 2019).

Dado exposto, os discursos das juventudes revelam uma perspectiva dos saberes científicos, compostos pela base da ciência moderna e suas práticas, hegemonicamente, centralizadas no corpo humano como templo de controle e disciplinamento para um funcionamento orgânico equilibrado.

Contudo, esses discursos também expõem nossas "ascese" como efeitos de poder e saber, mobilizados numa rede de comunicação interativa, produzindo novos ditos e não ditos, na iminência do conhecimento pelos modos de subjetivação das juventudes que, no contexto da educação em saúde, fabricam outras "asceses" na cotidianidade da vida social (Torres et al., 2020).

\section{Conclusão}

Os discursos juvenis apontam que os saberes das ciências modernas são fundantes aos modos de dizer e ver o corpo, ao passo que o processo de saúde e doença integra o conjunto de potências nas relações sociais. Todavia, estes saberes, que também são discursos desejantes, desenham perspectivas geográficas de uso das tecnologias digitais como outros espaços virtuais de realidades e ampliam o acesso à informação e a comunicação, coletivamente, como um "external HD which is 
knowledge repositories" humanos no mundo contemporâneo.

Assim, a extensão da comunicação virtual, onde os discursos das juventudes tiveram um loci de produção, circulação, repercussão e reinvenção, borrando as linguísticas, sonoridades, estéticas e éticas do ciberespaço não se encerra em si mesmo, pois as possibilidades de continuidade dos diálogos sobre a saúde auditiva, como um campo de saber poder afeito aos peritos do conhecimento científico, atravessam e vazam pelos diversos canais abertos à participação dos navegantes da rede mundial de comunicação, interligada por computadores.

Contudo, o seguimento de trabalhos futuros com as juventudes possibilitará a análise das suas visibilidades e dizibilidades discursivas sobre a saúde auditiva, bem como outros temas, com mediação na web radio, pondo em análise os deslocamentos, agenciamentos, regulações e atualizações dos saberes imanentes, mobilizados como prática de web cuidado com a vida humana.

\section{Referências}

Andrade, S. R., Schmitt, M. D., Storck, B. C., Piccoli, T., \& Ruoff, A. B. (2018). Análise Documental nas teses de enfermagem: técnica de coleta de dados e método de pesquisa. Cogitare Enfermagem, 23(1), 1-10. http://dx.doi.org/10.5380/ce.v23i1.53598.

Costa, S. R. S., Duqueviz, B. C., \& Pedroza, R. L. S. (2015). Tecnologias Digitais como instrumentos mediadores da aprendizagem dos nativos digitais. Psicol. Esc. Educ., 19(3), 603-610. http://dx.doi.org/10.1590/2175-3539/2015/0193912.

Ferreira, A. C. S., \& Freitas, G. M. S. (2018). O rádio na plataforma digital: A Web Rádio da Universidade Estadual da Paraíba e sua contribuição social e acadêmica. XX Congresso de Ciências da Comunicação na Região Nordeste. http://portalintercom.org.br/anais/nordeste2018/resumos/R62-0314-1.pdf.

Foucault, M. (1989). Microfísica do poder (8 ed). Graal.

Gonçalves, C. G. O., \& Fontoura, F. P. (2018). Intervenções educativas voltadas à prevenção de perda auditiva no trabalho: uma revisão integrativa. Rev. bras. saúde ocup, 43(supl. 1), 1-13. http://dx.doi.org/10.1590/2317-6369000032417.

Jacob, L. M. S., Melo, M. C., Sena, R. M. C., Silva, I. J., Mafetoni, R. R., \& Souza, K. C. S. (2019). Ações educativas para promoção da saúde na escola: revisão integrativa. Saúde e Pesquisa, 12(2), 419-426. https://doi.org/10.17765/2176-9206.2019v12n2p419-427.

Jardim, D. S., Maciel, F. J., Piastrelli, M. T., \& Lemos, S. M. A. (2017). Atenção à saúde auditiva: percepção dos usuários de um serviço público. CoDAS, 29(2), 1-7. https://doi.org/10.1590/2317-1782/20172015259.

Karimi, K. F. A., \& Nasiri, O. M. S. (2010). Noise induced hearing loss risk assessment in truck drivers. Noise Health., 12(46), 49-55. https://doi.org/10.4103/1463-1741.59999.

Keppler, H., Dhooge, I., \& Vinck, B. (2015). Hearing in young adults. Part II: The effects of recreational noise exposure. Noise Health., 17(78), 245-252. Disponível em: https://www.noiseandhealth.org/text.asp?2015/17/78/245/165026.

Nunes, A. D. S., Silva, C. R. L., Balen, A. S., Souza, D. L. B., \& Barbosa, I. R. (2019). Prevalência de deficiência auditiva e fatores associados em adolescentes e crianças em idade escolar: uma revisão sistemática. Braz. j. otorhinolaryngol., 85(2), 244-253. https://doi.org/10.1016/j.bjorl.2018.10.009.

Oliveira, M. F. F., Andrade, K. C., Carnaúba, A. T. L., Peixoto, G., \& Menezes, P. L. (2017). Fones de ouvido supra-aurais e intra-aurais: um estudo das saídas de intensidade e da audição de seus usuários. Audiology Comunication Research., 22, 1-8. https://doi.org/10.1590/2317-6431-2016-1783.

Oliveira, R. C., Santos, J. N., Rabelo, A. T. V., \& Magalhães, M. C. (2015). O impacto do ruído em trabalhadores de Unidades de Suporte Móveis. CoDAS, 27(3), 215-222. https://doi.org/10.1590/2317-1782/20152014136.

Pereira, A. C., \& Teles, M. J. (2013). Eficácia dos cerumenolíticos - uma revisão baseada na evidência para cuidados primários. Rev Port Med Geral Fam, 29, 172-8. Disponível em: http://www.scielo.mec.pt/pdf/rpmgf/v29n3/v29n3a05.pdf.

Rocha, C. H., Santos, L. H. D., Moreira, R. R., Neves-lobo, I. F., \& Samelli, A. G. (2011). Verificação da efetividade de uma ação educativa sobre proteção auditiva para trabalhadores expostos a ruído. J Soc Bras Fonoaudiol., 23(1), 38-43. https://doi.org/10.1590/S2179-64912011000100010.

Sales, C. V., \& Vasconcelos, M. A. D. M. (2016). Ensino Médio Integrado e Juventudes: desafios e projetos de futuro. Educ. Real., 41(1), 9-90. https://doi.org/10.1590/2175-623656094.

Santaella L. (2014). A aprendizagem ubíqua na educação aberta. Revista Tempos e Espaços em Educação, 7(14), 15-22. https://doi.org/10.20952/revtee.v0i0.3446.

Santos, F., \& Silva, J. P. (2019). Ansiedade entre as pessoas surdas: um estudo teórico. Arq. bras. Psicol., 71(1), 143-157. http://dx.doi.org/10.36482/18095267.ARBP2019v71i1p.143-157.

Silva, L. O., Noriega, J. A. V., \& Freire, H. B. G. (2018). Adaptação da escala de coping de Billings e Moos (ECBM) para surdos: um estudo piloto. Revista eletrónica de Psicología Iztalaca., 21(2). http://www.revistas.unam.mx/index.php/repi/article/view/65285. 
Research, Society and Development, v. 10, n. 7, e48810716776, 2021

(CC BY 4.0) | ISSN 2525-3409 | DOI: http://dx.doi.org/10.33448/rsd-v10i7.16776

Torres, R. A., Silva, M. A. M., Bezerra, A. E. M., Abreu, L. P. D., \& Mendonça, G. M. M. (2015). Comunicação em saúde: uso de uma web-rádio com escolares. Journal of Health Informatics, 2(7), 58-61.: http://www.jhi-sbis.saude.ws/ojs-jhi/index.php/jhi-sbis/article/view/325/233.

Torres, R. A. M., Freitas, G. H., Vieira, D. V. F., Torres, A. L. M. M., \& Silva, L. M. S. (2012). Tecnologias Digitais e Educação em Enfermagem: a utilização de uma Web-Rádio como estratégia pedagógica. Journal of Health Informatics., 4, 152-156. http://www.jhi-sbis.saude.ws/ojs-jhi/index.php/jhisbis/article/view/248.

Torres, R. A. M., Veras, K. C. B. B., Araújo, A. F., Gomes, K. V. L., Abreu, L. D. P., Gomes, E. D. P., Costa, I. G., \& Moraes, L. M. O. (2020). Knowledge of young schoolchildren about coronavirus via web radio: connections with the florence nightingale environmental theory. BJD., 6(11). https://www.brazilianjournals.com/index.php/BRJD/article/view/20303.

Vieira, G. I., Mendes, B. C. A., Zupelari, M. M., \& Pereira, I. M. T. B. (2015). Saúde auditiva no Brasil: análise quantitativa do período de vigência da Política Nacional de Atenção à Saúde Auditiva. Distúrb Comum, 27(4), 725-740. https://revistas.pucsp.br/index.php/dic/article/view/23915/18806.

Widén, S. E., Moller, C., \& Kahari, K. (2017). Headphone listening habits, hearing thresholds and listening levels in Swedish adolescents with severe to profound HL and adolescents with normal hearing. Int J Audiol, 57(10), 730-736. 10.1080/14992027.2018.1461938. 